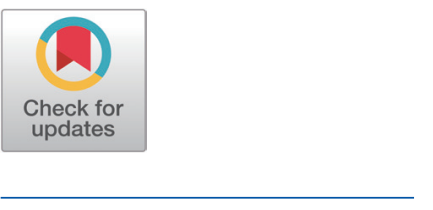

Received: Sep 30, 2021

Revised: Oct 13, 2021

Accepted: Oct 14, 2021

${ }^{*}$ Corresponding author

Seung Hwan Lee

Division of Animal and Dairy Science,

Chungnam National University,

Daejeon 34134, Korea.

Tel: +82-42-821-5772

E-mail: slee46@cnu.ac.kr

Copyright $\odot 2021$ Korean Society of

Animal Sciences and Technology.

This is an Open Access article

distributed under the terms of the

Creative Commons Attribution

Non-Commercial License (http://

creativecommons.org/licenses/by-

$\mathrm{nc} / 4.0 /$ ) which permits unrestricted

non-commercial use, distribution, and

reproduction in any medium, provided

the original work is properly cited.

ORCID

DooHo Lee

https://orcid.org/0000-0002-2174-7897

Yeongkuk Kim

https://orcid.org/0000-0002-6530-2304

Yoonji Chung

https://orcid.org/0000-0002-6906-6468

Dongjae Lee

https://orcid.org/0000-0002-8140-014X

Dongwon Seo

https://orcid.org/0000-0003-0548-7068

Tae Jeong Choi

https://orcid.org/0000-0002-8299-9342

Dajeong Lim

https://orcid.org/0000-0003-3966-9150

Duhak Yoon

https://orcid.org/0000-0002-3983-9757

\title{
Accuracy of genotype imputation based on reference population size and marker density in Hanwoo cattle
}

\author{
DooHo Lee ${ }^{1}$, Yeongkuk Kim¹, Yoonji Chung ${ }^{1}$, Dongjae Lee ${ }^{1}$, Dongwon Seo ${ }^{1}$, \\ Tae Jeong Choi ${ }^{2}$, Dajeong Lim ${ }^{3}$, Duhak Yoon ${ }^{4}$ and Seung Hwan Lee ${ }^{1 *}$ \\ ${ }^{1}$ Division of Animal and Dairy Science, Chungnam National University, Daejeon 34134, Korea \\ ${ }^{2}$ National Institute of Animal Science, Cheonan 31000, Korea \\ ${ }^{3}$ Animal Genomics and Bioinformatics Division, National Institute of Animal Science, Wanju 55365, Korea \\ ${ }^{4}$ Department of Animal Science \& Biotechnology, Kyungpook National University, Sangju 37224, Korea
}

\section{Abstract}

Recently, the cattle genome sequence has been completed, followed by developing a commercial single nucleotide polymorphism (SNP) chip panel in the animal genome industry. In order to increase statistical power for detecting quantitative trait locus (QTL), a number of animals should be genotyped. However, a high-density chip for many animals would be increasing the genotyping cost. Therefore, statistical inference of genotype imputation (low-density chip to high-density) will be useful in the animal industry. The purpose of this study is to investigate the effect of the reference population size and marker density on the imputation accuracy and to suggest the appropriate number of reference population sets for the imputation in Hanwoo cattle. A total of 3,821 Hanwoo cattle were divided into reference and validation populations. The reference sets consisted of $50 \mathrm{k}(38,916)$ marker data and different population sizes $(500,1,000,1,500,2,000$, and 3,600). The validation sets consisted of four validation sets (Total 889$)$ and the different marker density $(5 k[5,000], 10 k[10,000]$, and $15 k[15,000])$. The accuracy of imputation was calculated by direct comparison of the true genotype and the imputed genotype. In conclusion, when the lowest marker density (5k) was used in the validation set, according to the reference population size, the imputation accuracy was 0.793 to 0.929 . On the other hand, when the highest marker density (15k), according to the reference population size, the imputation accuracy was 0.904 to 0.967 . Moreover, the reference population size should be more than 1,000 to obtain at least $88 \%$ imputation accuracy in Hanwoo cattle.

Keywords: Single nucleotide polymorphism (SNP), Imputation, Hanwoo cattle, Reference population size, Marker density

\section{INTRODUCTION}

The complete cattle genome has been sequenced, and Illumina (San Diego, CA, USA) and Affymetrix (Santa Clara, CA, USA) have developed commercial single nucleotide polymorphism (SNP) chips that 
Seung Hwan Lee

https://orcid.org/0000-0003-1508-4887

Competing interests

No potential conflict of interest relevant to

this article was reported.

Funding sources

This study was supported by Research Funding (2020-0534-01) of Chungnam National University.

\section{Acknowledgements}

This work was supported by a grant and genomic data for imputation from the

BioGreen 21 Program(No. 015658022021),

Rural Development Administration, Korea.

Availability of data and material Upon reasonable request, the datasets of this study can be available from the corresponding author.

\section{Authors' contributions}

Conceptualization: Lee SH.

Data curation: Chung Y, Lee D, Lee SH.

Formal analysis: Lee DH, Kim Y Lee SH.

Methodology: Lee DH, Seo D, Lee SH.

Software: Lee DH, Choi TJ, Lim D.

Validation: Lee DH, Yoon D.

Investigation: Lee $\mathrm{DH}$, Lee SH

Writing - original draft: Lee DH, Lee SH.

Writing - review \& editing: Lee $\mathrm{DH}$, Chung

Y, Lee D, Seo D, Choi TJ, Lim D, Yoon D, Lee SH.

Ethics approval and consent to participate This article does not require IRB/IACUC approval because there are no human and animal participants. use chip-based array technology [1]. The development of SNP panels has enabled many studies, such as genome-wide association studies (GWAS) and best linear unbiased prediction (BLUP) studies [2]. Many genetic markers associated with objective breeding traits have been identified for marker-assisted selection [3]. Using a high-density SNP panel in a GWAS increases the probability of finding quantitative trait locus regions [4]. Improved high-density SNP panels also increase the accuracy of genomic breeding value estimations using genomic BLUP [5-8].

However, it is very difficult to genotype all animals in a population because of the cost of highdensity chips. In addition, SNP panels for different platforms, which may differ in density or chip data versions, are not completely compatible. Imputation methods for converting from low- to high-density data are an alternative [9].

Genotype imputation refers to statistical inference of genotype and includes family and population-based methods. Family based methods require sufficient pedigree information to compare reference and test groups, so are difficult to apply when there is no pedigree information or insufficient pedigree depth [10,11]. Population-based methods predict low-density genotypes of animals by referring to a reference population genotyped at high density. This method uses a library and haplotype clustering to find the most appropriate haplotype and genotype [12-15]. Many factors affect the imputation accuracy of this method, including the reference population size, relationship between animals in the reference and test populations, minor allele frequency of the SNP to be imputed, proportion of missing genotypes on the low- and high-density panels, marker density, population structure, and the level of linkage disequilibrium (LD) [16]. Generally, family based methods aim to identify the animals to be sequenced, while population-based methods aim at imputation of the genotypes of unrelated individuals. Many studies have examined ways to increase imputation accuracy using population-based imputation software, such as fastPHASE [17], Beagle [18], Minimac [19], and findhap.f90 [20]. However, no studies have examined imputation accuracy in Hanwoo cattle according to the reference population size and marker density. Hanwoo cattle is a native taurine cattle breed in Korea and has been bred as a draft animal since 5,000 years ago. Over time, Hanwoo cattle have been bred for meat production and have become very popular despite high prices due to marbling fat, softness, juiciness, and unique flavor. [21]

Therefore, this study investigated the efficacy of genotyping by imputation of a high-density chip from a low-density one, according to the reference population size and marker density, and proposes an appropriate reference population size for high-quality imputation in Hanwoo cattle.

\section{MATERIALS AND METHODS}

\section{Genotypes data}

All the data-set (50K genotypes) used in this study was provided from the previous Research Project (BioGreen21, Hanwoo Research Institutes of National Institute of Animal Science, RDA) and current research project (Bridge Project of NIAS, RDA). To investigated imputation accuracy, the 3,821 animals were randomly selected from the population.

\section{Quality control}

Genotype data was modified using GenomeStudio (Illumina) ver. 2.0 software with a genotyping module to fit the analysis software format: Illumina data file (.bsc) to genotype file format (.ped). We removed SNPs in unknown chromosomes and sex chromosomes for the next steps. The quality control procedure was performed using plink1.9 software [22]. The raw data has a $95.55 \%$ genotyping rate, so missing genotype data phasing was performed as a pre-imputation task for the imputation accuracy as a reference population. SNP data were subjected to strict quality control to 
minimize the impact of the imputation accuracy on genotyping error: minor allele frequency (0.01), genotyping call rate (0.9), missing individuals (0.1), Hardy-Weinberg equilibrium test $p$-value (0.0001). After quality control, a total of 38,933 SNPs were used for analysis. the number of SNPs on each chromosome before and after quality control is described in Table 1.

\section{Imputation scenarios}

Imputation scenarios are set based on population size and marker density. The population size controls the size of the reference population, and the marker density controls the maker density of the test population. The test population was selected by the lowest birth year belonging to the data set. Thus, the test population consists of 889 animals, and these were divided into four validation sets. In the reference population, five reference populations were constructed. First, 500, 1,000,

Table 1. Number of SNPs on each chromosomes between before and after in quality control

\begin{tabular}{|c|c|c|c|}
\hline \multirow{2}{*}{ Chromosome } & Before QC & After QC & \multirow{2}{*}{$\begin{array}{l}\text { Removed } \\
\text { No. SNP }\end{array}$} \\
\hline & No. SNP & No. SNP & \\
\hline 1 & 3,133 & 2,567 & 566 \\
\hline 2 & 2,553 & 2,037 & 516 \\
\hline 3 & 2,279 & 1,905 & 374 \\
\hline 4 & 2,357 & 1,901 & 456 \\
\hline 5 & 2,050 & 1,611 & 439 \\
\hline 6 & 2,373 & 1,980 & 393 \\
\hline 7 & 2,141 & 1,725 & 416 \\
\hline 8 & 2,181 & 1,786 & 395 \\
\hline 9 & 1,899 & 1,541 & 358 \\
\hline 10 & 1,977 & 1,618 & 359 \\
\hline 11 & 2,058 & 1,666 & 392 \\
\hline 12 & 1,599 & 1,280 & 319 \\
\hline 13 & 1,666 & 1,370 & 296 \\
\hline 14 & 1,687 & 1,386 & 301 \\
\hline 15 & 1,583 & 1,255 & 328 \\
\hline 16 & 1,542 & 1,222 & 320 \\
\hline 17 & 1,442 & 1,189 & 253 \\
\hline 18 & 1,249 & 1,001 & 248 \\
\hline 19 & 1,274 & 1,047 & 227 \\
\hline 20 & 1,408 & 1,144 & 264 \\
\hline 21 & 1,313 & 1,048 & 265 \\
\hline 22 & 1,194 & 957 & 237 \\
\hline 23 & 976 & 817 & 159 \\
\hline 24 & 1,209 & 990 & 219 \\
\hline 25 & 905 & 747 & 158 \\
\hline 26 & 1,012 & 810 & 202 \\
\hline 27 & 895 & 727 & 168 \\
\hline 28 & 889 & 730 & 159 \\
\hline 29 & 966 & 796 & 170 \\
\hline 30 & 1,044 & 80 & 964 \\
\hline Total & 48,854 & 38,933 & 9,921 \\
\hline
\end{tabular}

SNP, single nucleotide polymorphism; QC, quality control. 
1,500, and 2,000 animals are selected based on the individuals not included in the test population. In addition, 2,000 animals and the remaining test populations not included in each validation were included as reference groups to constitute over $2,000(3,600)$ reference groups. When increasing the number of reference populations, the first 500 animals were randomly selected from the data set using the R program ver 3.6 [23], and another 500 animals were added from the remaining individuals. Thus, each scenario set is described in Table 2. and Fig. 1. In addition, three low-density SNP panels are created to use for validation marker density. Each SNP panel selected evenly spaced $5 \mathrm{k}, 10 \mathrm{k}$, and 15k from a 50k Illumina chip, and the number and average distance of SNPs for each chromosome of these panels are described in Table 3. The test population data were analyzed using the generated low-density SNP panel information. A schematic diagram of the imputation scenario is illustrated in Fig. 2.

\section{Linkage disequilibrium}

We analyzed the LD level, which is one of the factors affecting imputation accuracy. Because imputation uses haplotype information, imputation accuracy will decrease if the LD levels of the reference population and test population LD levels are different. LD value $\left(r^{2}\right)$ between SNPs within $1 \mathrm{Mb}$ distance was measured using plink1.9 software. This means that the maximum distance between the markers is $1 \mathrm{Mb}$, and the average $r^{2}$ value is estimated for each autosomal chromosome. The following formula is used for $\mathrm{LD}$ estimation [24].

Table 2. Summary of imputation scenarios using different reference population size and validation data set

\begin{tabular}{|c|c|c|c|c|c|c|c|c|c|c|c|c|}
\hline \multirow{3}{*}{$\begin{array}{l}\text { Scenario } \\
\text { set }\end{array}$} & \multirow{3}{*}{$\begin{array}{l}\text { Reference } \\
\text { SNP data }\end{array}$} & \multirow{2}{*}{\multicolumn{2}{|c|}{ Reference set }} & \multicolumn{8}{|c|}{ Test set } & \multirow{3}{*}{$\begin{array}{c}\text { Total } \\
\text { animal }\end{array}$} \\
\hline & & & & \multicolumn{2}{|c|}{ Validation 1} & \multicolumn{2}{|c|}{ Validation 2} & \multicolumn{2}{|c|}{ Validation 3} & \multicolumn{2}{|c|}{ Validation 4} & \\
\hline & & $\mathbf{N}$ & $\%$ & $\mathbf{N}$ & $\%$ & $\mathbf{N}$ & $\%$ & $\mathbf{N}$ & $\%$ & $\mathbf{N}$ & $\%$ & \\
\hline Ref 500 & $50 \mathrm{k}$ & 500 & 0.69 & 222 & 0.31 & 223 & 0.31 & 223 & 0.31 & 221 & 0.31 & $721-723$ \\
\hline Ref 1,000 & $50 \mathrm{k}$ & 1,000 & 0.82 & 222 & 0.18 & 223 & 0.18 & 223 & 0.18 & 221 & 0.18 & $1,221-1,223$ \\
\hline Ref 1,500 & $50 \mathrm{k}$ & 1,500 & 0.87 & 222 & 0.13 & 223 & 0.13 & 223 & 0.13 & 221 & 0.13 & $1,721-1,723$ \\
\hline Ref 2,000 & $50 \mathrm{k}$ & 2,000 & 0.90 & 222 & 0.10 & 223 & 0.10 & 223 & 0.10 & 221 & 0.10 & $2,221-2,223$ \\
\hline Ref $2,000+$ & $50 \mathrm{k}$ & 3,600 & 0.94 & 222 & 0.06 & 223 & 0.06 & 223 & 0.06 & 221 & 0.06 & 3,821 \\
\hline
\end{tabular}

Total animal, The total number of the reference population and validation animals; Ref, reference population; SNP, single nucleotide polymorphism.

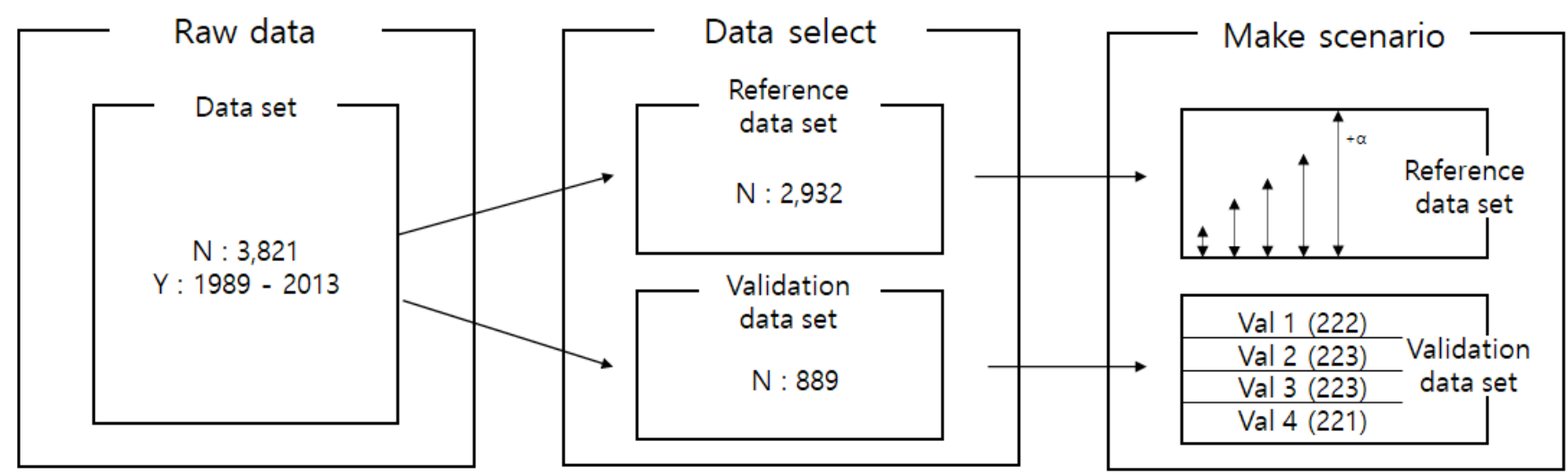

Fig. 1. Organizing or analyzing data. The raw data consisted of a total of 3,821 individuals born between 1989 and 2013. First, the youngest individuals in the dataset were selected as the validation set. Then, the others were configured as a reference group. The reference group was constructed by changing the group size and the validation group by changing the number of markers. Val, validation. 
Table 3. Each chromosome information about the number of SNP and average distance according to marker density

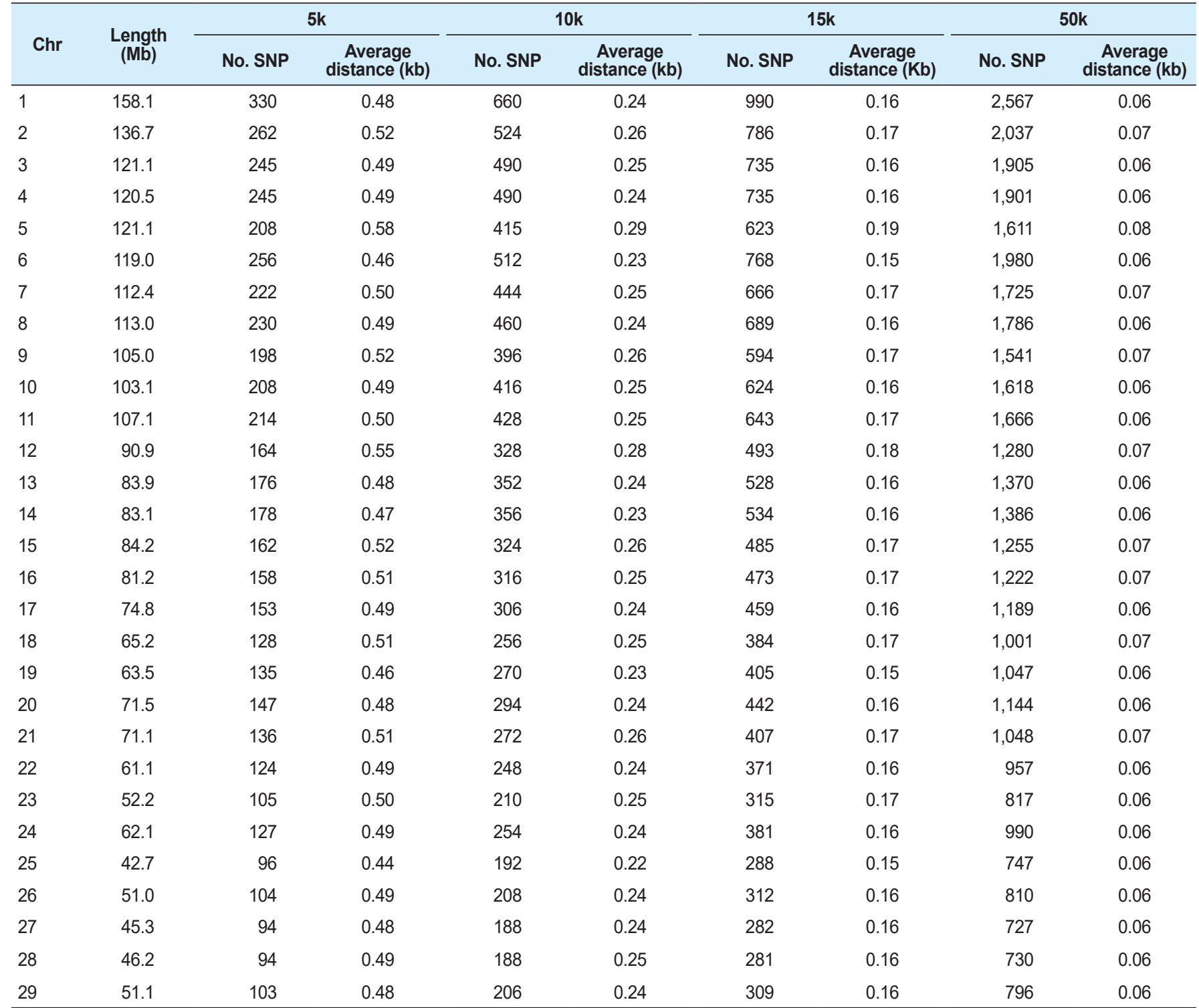

SNP, single nucleotide polymorphism.

$$
r^{2}=\frac{\left(p_{A 1 B 1}-p_{A 1} p_{B 1}\right)^{2}}{p_{A 1} p_{A 2} p_{B 1} p_{B 2}}
$$

Where, $\mathrm{A} 1, \mathrm{~A} 2, \mathrm{~B} 1$, and $\mathrm{B} 2$ are the alleles of SNP $\mathrm{A}$ and $\mathrm{SNP} \mathrm{B}$, and $\mathrm{P}_{\mathrm{A} 1}, \mathrm{P}_{\mathrm{A} 2}, \mathrm{P}_{\mathrm{B} 1}$, and $\mathrm{P}_{\mathrm{B} 2}$ are the corresponding allele frequencies. $\mathrm{P}_{\mathrm{A} 1 \mathrm{~B} 1}$ is the haplotype frequency of A1B1. The average LD levels of the reference and test populations used in all scenarios were analyzed whether they showed a similar pattern according to the SNPs' distance. Also, the LD pattern was investigated in the test population according to the marker density $(5 \mathrm{k}, 10 \mathrm{k}, 15 \mathrm{k}$, and $50 \mathrm{k})$.

\section{Genotype imputation}

Imputation of low-density (5k, 10k, 15k) data set to high-density (50k) genotypes was performed with the beagle program ver. 3.3 [25]. The beagle program, which is a population-based method, does not require pedigree information. The beagle program clusters haplotypes in each marker using 


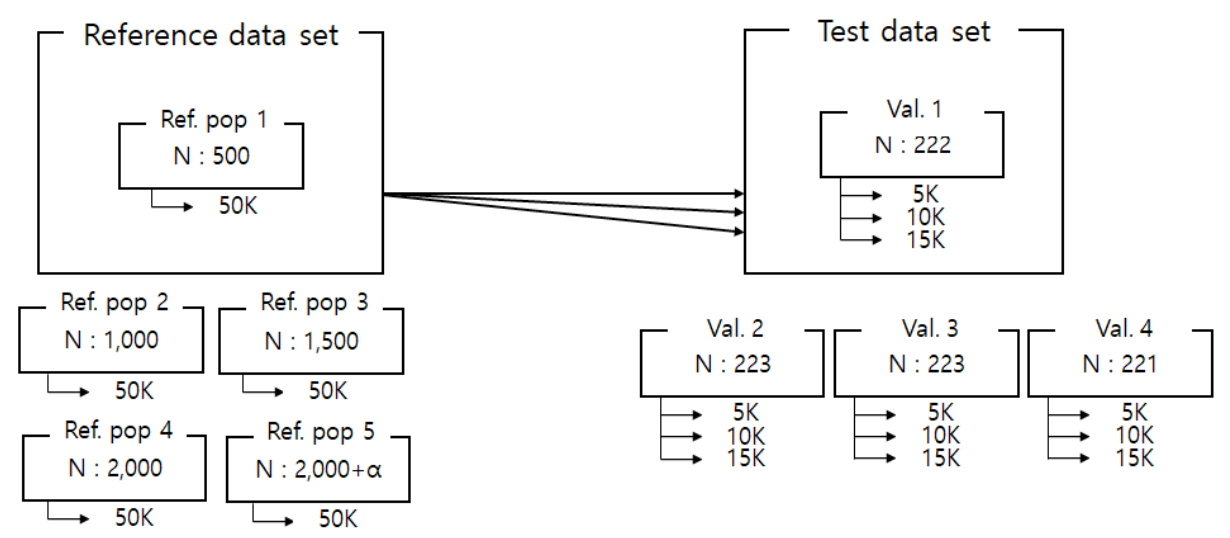

Fig. 2. Imputation scenarios. This figure shows reference population 1 and validation 1 as examples, and imputation was performed on each marker density (5k,10k, and 15k). Since the reference data set consists of 5 groups, and the test set consists of 4 validation groups, the imputation process ran 60 times. Ref, reference Val, validation.

a localized haplotype cluster model and then uses the Hidden Markov Model (HMM) to find the most probable haplotype based on the known genotype of each individual [26]. Therefore, collecting haplotype information and imputing un-genotyped SNP in the reference population is important for imputing validation data from low-density to high-density. The imputation was performed for each chromosome by pairing the reference data set and the validation data set in all scenarios. After imputation, the genotype was recorded for accuracy comparison, the $\mathrm{AA}, \mathrm{AB}$, and $\mathrm{BB}$ types were changed to 0,1 , and 2 , respectively. The ratio was used as the imputation accuracy by direct genotype comparison of raw genotype and imputed genotypes. In addition, how the imputation accuracy changes are checked according to the minor allele frequency, reference population size, and marker density.

\section{RESULTS}

\section{Linkage disequilibrium}

We investigated the LD pattern of all of our scenario sets (Fig. 3). Fig. 3A shows the LD pattern of the four validation sets according to marker density (5k,10k, and $15 \mathrm{k})$. In all validation sets, the overall LD estimation results showed very similar tendency; the level of LD decreases as the distance to the SNP increases. In each validation set, the LD pattern also differed slightly with the marker density, but the difference was less than 0.01 . As there was no difference among the validation sets, the data were free from bias. Fig. 3B shows the LD pattern of the reference sets according to population size. The LD levels of the reference sets used in all scenarios were similar when the reference population size was $500,1,000,1,500,2,000$, or 3,600 . Because the population size is larger than the validation set, the $\mathrm{LD}$ level does not change as the population size changes relative to the reference population. In addition, the LD level difference among the reference groups was smaller than the LD level difference among the validation sets. Comparing the validation set with the reference population set showed that the $\mathrm{LD}$ levels have similar patterns. In particular, the distance between SNPs can be divided into 0-20, 20-50, 50-100, 100-200, 200-500, 500-1,000 $\mathrm{kb}$. Table 4 gives the number of SNP pairs in the reference and validation sets, and the average r-square $\left(r^{2}\right)$ values and deviations. As the distance between SNPs increased, the number of SNP pairs gradually increased; there were 750 pairs at $0-20 \mathrm{~kb}$ and 500,000 at 200-1,000 $\mathrm{kb}$. The $r^{2}$ value was $0.28(0.32)$ at $0-20 \mathrm{~kb}$ and $0.02(0.04)$ at $200-1,000 \mathrm{~kb}$; it decreased rapidly up to the first 200 
A

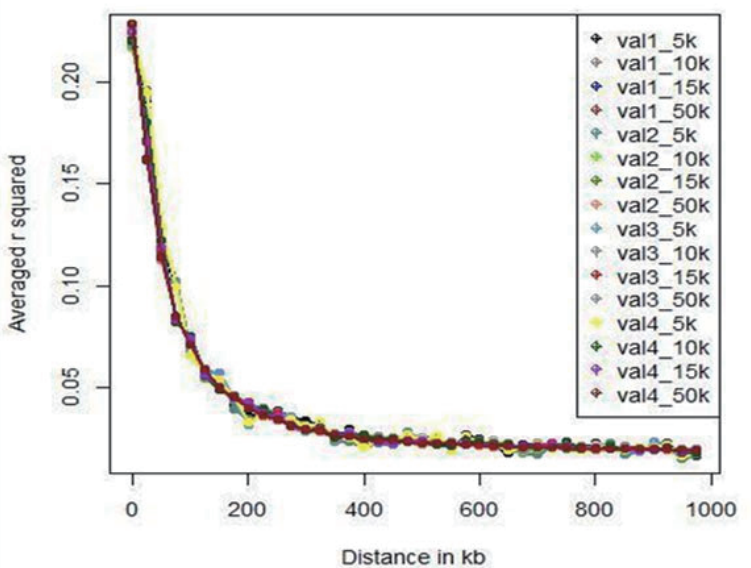

B

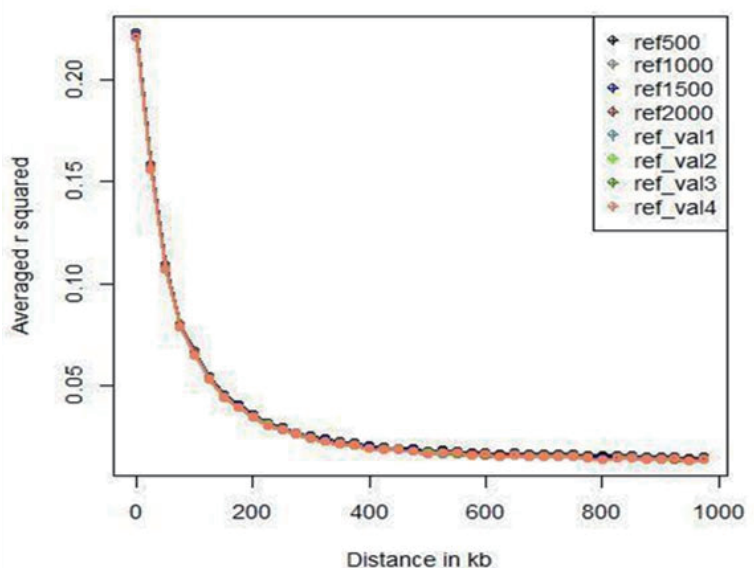

Fig. 3. The interval means linkage disequilibrium $\left(r^{2}\right)$ value between marker pairs about the marker distance according to the test set $(A)$ and reference set (B). (A) Total four validation data sets have different marker density consisted of 5k, 10k, 15k, and 50k for imputation. (B) Total five reference data sets consisted of 500,1,000,1,500,2,000, and over 2,000 (3,600). In addition, over 2,000 reference data include other validation data also into reference data.

Table 4. Linkage disequilibrium $\left(r^{2}\right)$ information in all scenario data sets

\begin{tabular}{|c|c|c|c|c|c|c|c|c|c|c|}
\hline \multirow[b]{2}{*}{ Data type } & \multicolumn{2}{|c|}{ 0-20 kb interval } & \multicolumn{2}{|c|}{ 20-50 kb interval } & \multicolumn{2}{|c|}{$50-100 \mathrm{~kb}$ interval } & \multicolumn{2}{|c|}{$100-200 \mathrm{~kb}$ interval } & \multicolumn{2}{|c|}{$200-1,000$ kb interval } \\
\hline & $\begin{array}{c}\text { No. SNP } \\
\text { pair }\end{array}$ & $\begin{array}{c}\text { Average } r^{2} \\
\text { (SD) }\end{array}$ & $\begin{array}{c}\text { No. SNP } \\
\text { pair }\end{array}$ & $\begin{array}{c}\text { Average } r^{2} \\
\text { (SD) }\end{array}$ & $\begin{array}{l}\text { No. SNP } \\
\text { pair }\end{array}$ & $\begin{array}{c}\text { Average } r^{2} \\
\text { (SD) }\end{array}$ & $\begin{array}{l}\text { No. SNP } \\
\text { pair }\end{array}$ & $\begin{array}{l}\text { Average } r^{2} \\
\text { (SD) }\end{array}$ & $\begin{array}{c}\text { No. SNP } \\
\text { pair }\end{array}$ & $\begin{array}{l}\text { Average } r^{2} \\
\text { (SD) }\end{array}$ \\
\hline Val 1 & 750 & $0.28(0.33)$ & 22,732 & $0.17(0.25)$ & 33,014 & $0.10(0.17)$ & 64,732 & $0.06(0.11)$ & 500,518 & $0.02(0.04)$ \\
\hline Val 2 & 751 & $0.28(0.33)$ & 22,722 & $0.17(0.25)$ & 33,002 & $0.10(0.17)$ & 64,713 & $0.06(0.11)$ & 500,429 & $0.02(0.04)$ \\
\hline Val 3 & 751 & $0.28(0.33)$ & 22,731 & $0.17(0.25)$ & 33,010 & $0.10(0.17)$ & 64,732 & $0.06(0.11)$ & 500,464 & $0.02(0.04)$ \\
\hline Ref 500 & 744 & $0.27(0.32)$ & 22,572 & $0.17(0.24)$ & 32,793 & $0.09(0.16)$ & 64,243 & $0.05(0.10)$ & 496,752 & $0.02(0.04)$ \\
\hline $\operatorname{Ref} 1,000$ & 749 & $0.27(0.32)$ & 22,604 & $0.17(0.24)$ & 32,814 & $0.09(0.16)$ & 64,299 & $0.05(0.10)$ & 497,219 & $0.02(0.04)$ \\
\hline Ref 1,500 & 749 & $0.27(0.32)$ & 22,618 & $0.17(0.24)$ & 32,827 & $0.09(0.16)$ & 64,374 & $0.05(0.10)$ & 497,641 & $0.02(0.04)$ \\
\hline Ref 2,000 & 750 & $0.27(0.32)$ & 22,646 & $0.17(0.24)$ & 32,877 & $0.09(0.16)$ & 64,488 & $0.05(0.10)$ & 498,467 & $0.02(0.04)$ \\
\hline $\operatorname{Ref} 2,000+($ val 4$)$ & 752 & $0.27(0.32)$ & 22,739 & $0.17(0.24)$ & 33,025 & $0.09(0.16)$ & 64,758 & $0.05(0.10)$ & 500,701 & $0.02(0.04)$ \\
\hline
\end{tabular}

SNP, single nucleotide polymorphism; Val, validation, Ref, reference population.

$\mathrm{kb}$, and decreased slowly thereafter.

Imputation accuracy

We assessed the genotype imputation accuracy according to the SNP panel density and reference population size. The average of the four validation sets represents the accuracy of each scenario. The lowest imputation accuracy was $79 \%$ with $5 \mathrm{k}$ marker density and a reference population of 500, and the highest accuracy was $97 \%$ with $15 \mathrm{k}$ marker density and a reference population over 2,000 $(3,600)$. When we assessed the accuracy of each validation set, the maximum difference among the sets was about $4 \%$, when the reference population size was 1,000 and the marker density $5 \mathrm{k}$. The other scenarios had similar imputation accuracies. Fig. 4 plots the accuracy according to the marker density of each chromosome for a reference population of 1,500 (The imputation accuracies for 


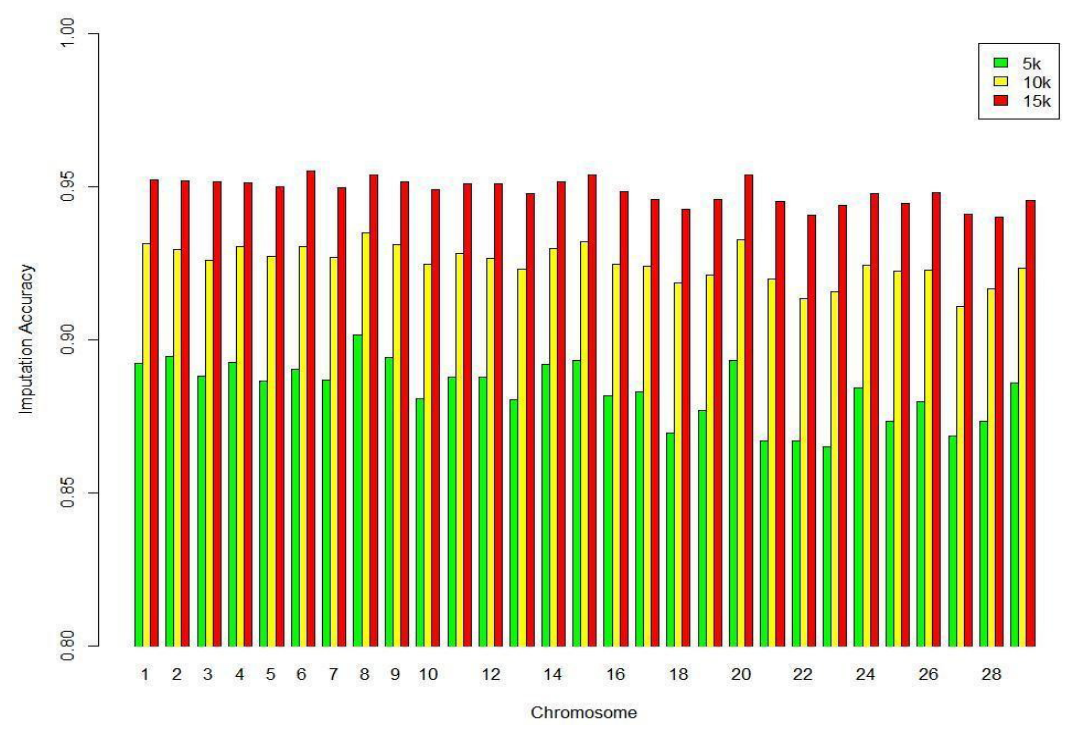

Fig. 4. Average imputation accuracy of each chromosome different marker density in reference population size 1,500 . The average accuracy of each chromosome is indicated by a different color depending on the marker density of the test data set, which is $5 \mathrm{k}, 10 \mathrm{k}, 15 \mathrm{k}$ represented to green, yellow, and red, respectively.

each chromosome for reference population sizes 500, 10,00, 2,000, and over 2,000 are presented in Figs. 5-8); chromosome 21 show maximum variability in imputation accuracy. Fig. 9 plots the misplaced SNPs on the entire autosomal segment with markers of $5 k(A), 10 k(B)$, and $15 k(C)$ from above and confirms the presence of several regions with poor imputation quality. Based on 0.75 as a threshold, 1275 SNPs were identified as substandard at $5 \mathrm{k}, 151$ SNPs at $10 \mathrm{k}$, and 65 SNPs were identified as substandard at $15 \mathrm{k}$.

\section{Imputation accuracy by marker density}

This study investigated the effect of marker density on imputation accuracy. Three low-density $(5 \mathrm{k}, 10 \mathrm{k}$, and $15 \mathrm{k})$ datasets were used as marker data for validation and imputed to high-density (50k; 38,933). In the 5k marker dataset, the imputation accuracy was $0.793-0.929$, with a $13.6 \%$

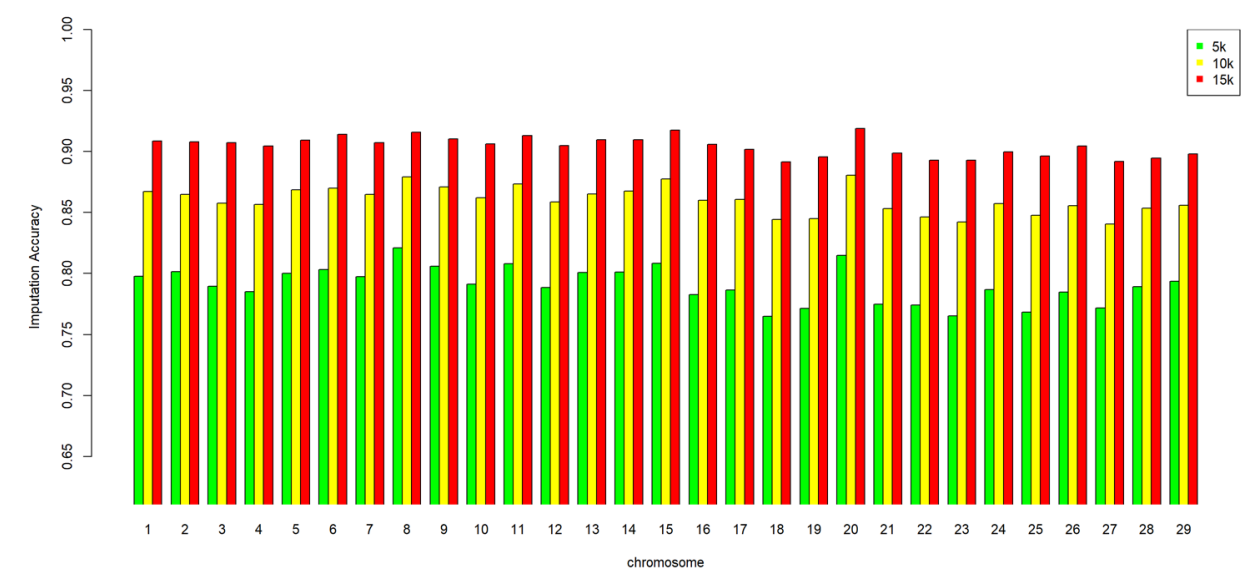

Fig. 5. Average imputation accuracy of each chromosome different marker density in reference population size 500 . The average accuracy of each chromosome is indicated by a different color depending on the marker density of the test data set, which is $5 \mathrm{k}, 10 \mathrm{k}, 15 \mathrm{k}$ represented to green, yellow, and red, respectively. 


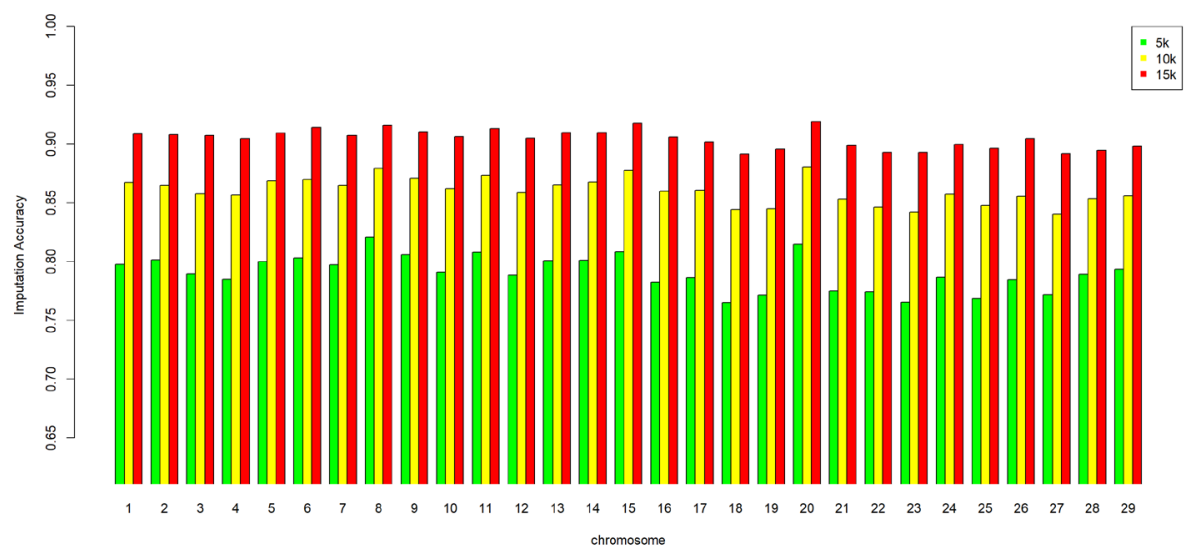

Fig. 6. Average imputation accuracy of each chromosome different marker density in reference population size 1,000 . The average accuracy of each chromosome is indicated by a different color depending on the marker density of the test data set, which is $5 \mathrm{k}, 10 \mathrm{k}, 15 \mathrm{k}$ represented to green, yellow, and red, respectively.

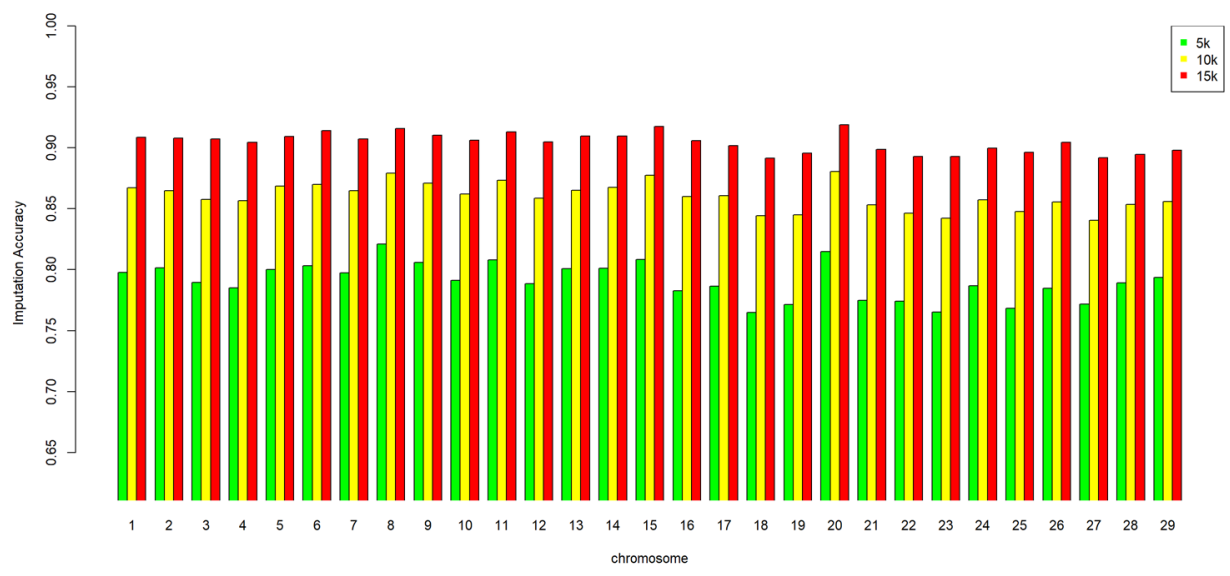

Fig. 7. Average imputation accuracy of each chromosome different marker density in reference population size 2,000. The average accuracy of each chromosome is indicated by a different color depending on the marker density of the test data set, which is $5 \mathrm{k}, 10 \mathrm{k}, 15 \mathrm{k}$ represented to green, yellow, and red, respectively.

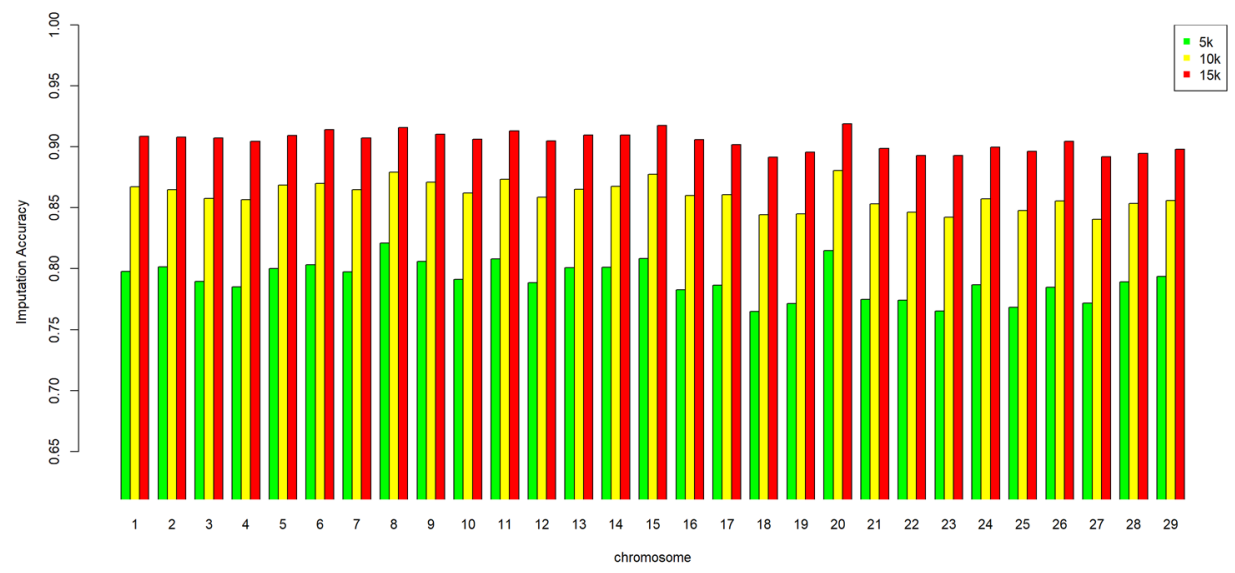

Fig. 8. Average imputation accuracy of each chromosome different marker density in reference population size over 2,000 . The average accuracy of each chromosome is indicated by a different color depending on the marker density of the test data set, which is $5 \mathrm{k}, 10 \mathrm{k}, 15 \mathrm{k}$ represented to green, yellow, and red, respectively. 


\section{(A)}

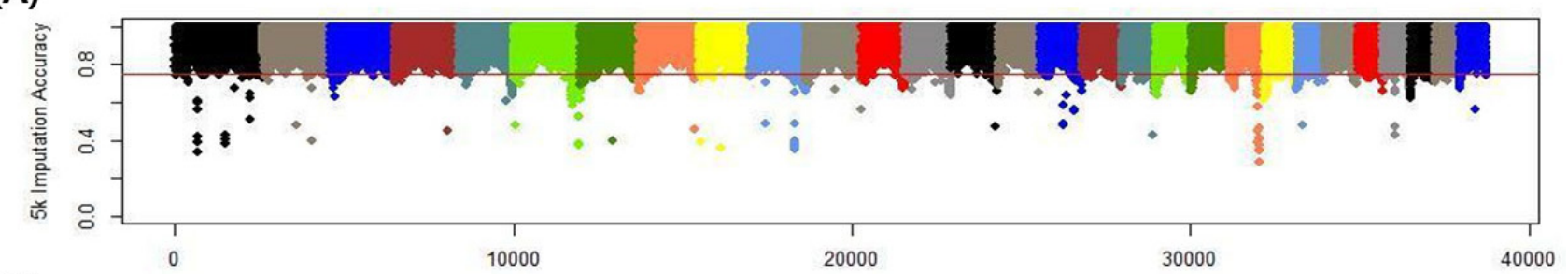

(B)

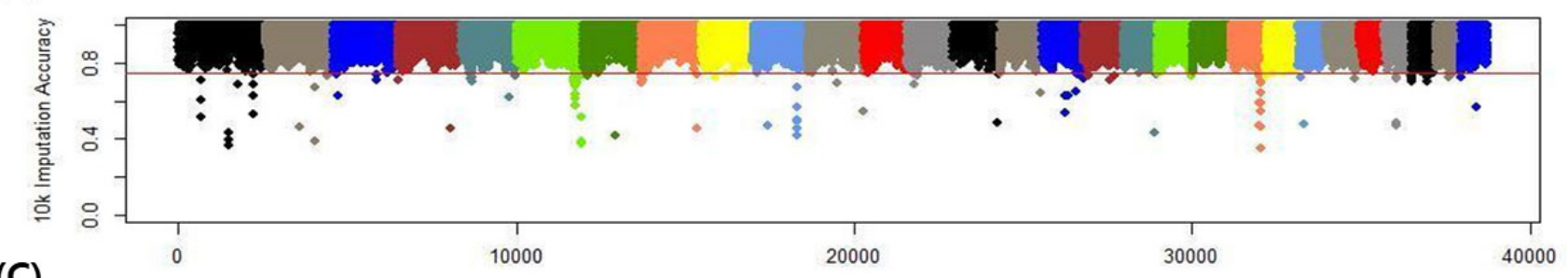

(C)

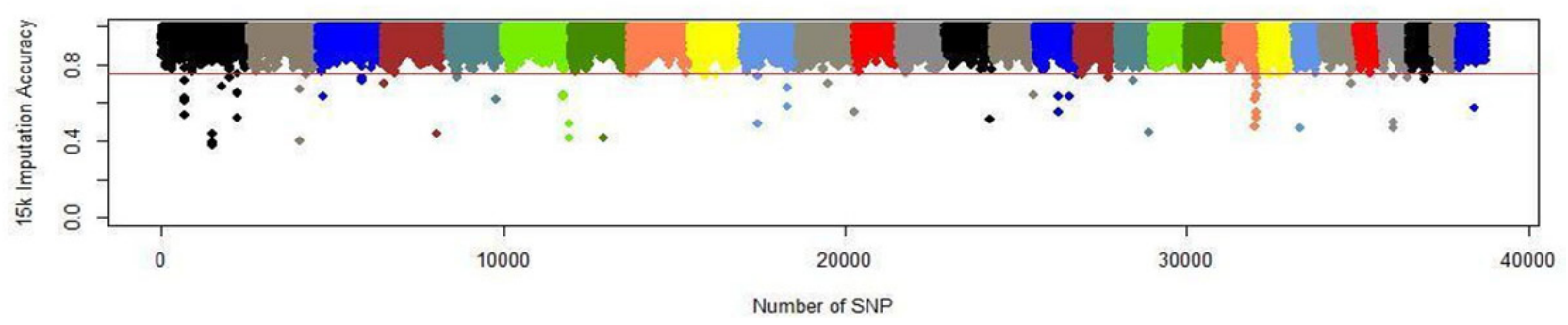

Fig. 9. Average imputation accuracy of each SNPs different marker density in reference population size 1,500. We did genome-wide plotting for each SNP imputation accuracy to find a region where the low imputation efficiency. Each chromosome has a different color, and the inferior area exists at the end of the chromosome. As the marker density increases; (A) 5k, (B) 10k and (C) 15k from above, the overall imputation accuracy also increases. 1,275 SNPs were identified as substandard at 5k, 151 SNPs at 10k, and 65 SNPs were identified as substandard at 15k. Brown horizontal threshold set to 0.75 . SNP, single nucleotide polymorphism.

accuracy difference according to the reference population size. In comparison, in the $15 \mathrm{k}$ marker dataset, the imputation accuracy was $0.904-0.967$, with a $6.3 \%$ accuracy difference according to the reference population size (Table 5). This shows that the higher the density of the validation set, the greater the imputation accuracy; moreover, the imputation accuracy difference according to reference population size is much greater at low than high density. The difference in imputation accuracy between $5 \mathrm{k}$ and $10 \mathrm{k}$ is also more significant than that between $10 \mathrm{k}$ and $15 \mathrm{k}$. The efficiency of imputation increased with marker density in the validation set. Imputation took a comparatively long time when the marker density of the validation set was low. Time costs are not shown in this study.

\section{Imputation accuracy by reference population size}

Five reference populations were examined: 500,1,000,1,500,2,000, and 3,600. When selecting the animals for the reference population, we used random sampling based on birth year; the relatedness of the animals was not considered. Fig. 10 plots the average imputation accuracy according to reference population size and test data marker density. For the smallest reference population ( $\mathrm{n}=$ 500 ), the imputation accuracy was $0.793-0.906$, differing by $11.3 \%$ according to marker density. For the largest reference population $(3,600)$, the imputation accuracy was $0.929-0.969$, differing by $4 \%$ according to marker density (Table 5). These results show that the larger the reference population, the higher the imputation accuracy. Moreover, the difference in imputation accuracy according to 
Table 5. Average imputation accuracy of validation data sets

\begin{tabular}{|c|c|c|c|c|c|c|c|}
\hline Density & No. Ref & Val 1 & Val 2 & Val 3 & Val 4 & Average & SD \\
\hline \multirow[t]{5}{*}{$5 k$} & 500 & 0.796 & 0.789 & 0.797 & 0.792 & 0.793 & 0.004 \\
\hline & 1,000 & 0.862 & 0.938 & 0.862 & 0.861 & 0.881 & 0.038 \\
\hline & 1,500 & 0.886 & 0.883 & 0.888 & 0.886 & 0.886 & 0.002 \\
\hline & 2,000 & 0.899 & 0.897 & 0.902 & 0.9 & 0.9 & 0.002 \\
\hline & $2,000+$ & 0.929 & 0.926 & 0.931 & 0.929 & 0.929 & 0.002 \\
\hline \multirow[t]{5}{*}{$10 \mathrm{k}$} & 500 & 0.864 & 0.859 & 0.864 & 0.861 & 0.862 & 0.002 \\
\hline & 1,000 & 0.909 & 0.907 & 0.911 & 0.91 & 0.909 & 0.002 \\
\hline & 1,500 & 0.927 & 0.924 & 0.928 & 0.927 & 0.926 & 0.001 \\
\hline & 2,000 & 0.936 & 0.934 & 0.938 & 0.937 & 0.936 & 0.002 \\
\hline & $2,000+$ & 0.955 & 0.953 & 0.956 & 0.955 & 0.955 & 0.001 \\
\hline \multirow[t]{5}{*}{$15 \mathrm{k}$} & 500 & 0.907 & 0.904 & 0.908 & 0.906 & 0.906 & 0.002 \\
\hline & 1,000 & 0.938 & 0.936 & 0.939 & 0.938 & 0.937 & 0.001 \\
\hline & 1,500 & 0.949 & 0.948 & 0.951 & 0.95 & 0.949 & 0.001 \\
\hline & 2,000 & 0.955 & 0.954 & 0.957 & 0.956 & 0.956 & 0.001 \\
\hline & $2,000+$ & 0.969 & 0.967 & 0.969 & 0.969 & 0.969 & 0.001 \\
\hline
\end{tabular}

Ref, reference Val, validation.

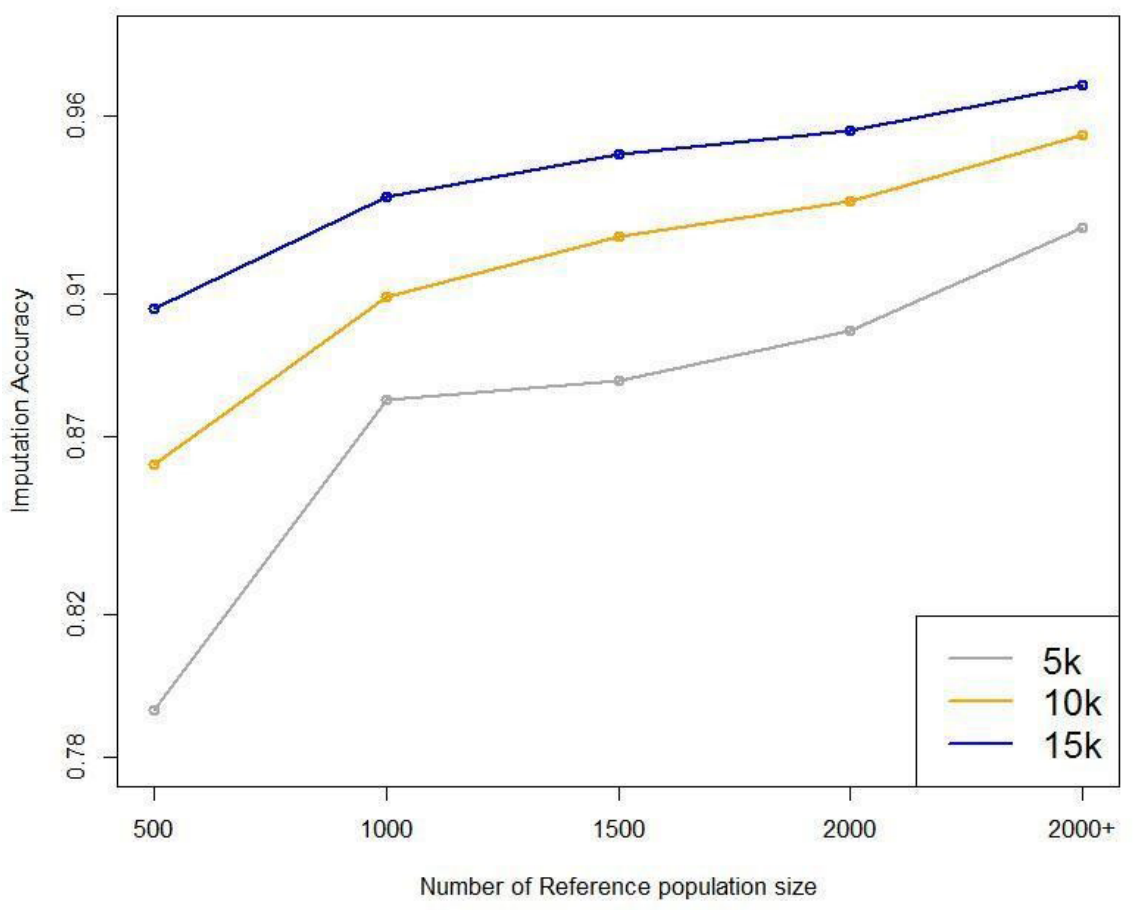

Fig. 10. The Average accuracy of imputation according to reference population size and validation data marker density. The validations average imputation accuracy was calculated according to the reference population size, which was displayed according to the density of each marker density. Gray, yellow, and blue represent $5 \mathrm{k}, 10 \mathrm{k}$, and $15 \mathrm{k}$, respectively.

marker density is much more significant with small reference populations. When the reference population in Hanwoo cattle exceeds 1,000 , the average imputation accuracy exceeds $88 \%$, even using $5 \mathrm{k}$ SNP data (Fig. 10). The imputation efficiency increased with reference population size, and imputation took longer if the reference population was small. 


\section{Imputation accuracy by minor allele frequency}

To investigate the effect of minor allele frequency, which affects imputation accuracy, the minor allele frequency of all SNPs was increased from 0 to 0.5 in 0.005 increments, with 100 groups in all scenarios. The imputation accuracy of each minor allele group based on population size and marker density was compared. Fig. 11 shows the imputation accuracy in five reference populations with three different marker densities up to $50 \mathrm{k}$, according to the minor allele frequencies. The imputation accuracy was negatively related to the minor allele frequency, confirming that the imputation accuracy decreased as the minor allele frequency increased. Using the $5 \mathrm{k}$ marker data in the validation set, the 0.005 and 0.5 groups had accuracies of $98.3 \%-99.4 \%$ and $69.4 \%-88.9 \%$, respectively, depending on the size of the reference group, and the difference in accuracy was $10.5 \%-28.9 \%$. However, when the $15 \mathrm{k}$ marker data was used in the validation set, the 0.005 and 0.5 groups had respective accuracies of $99.3 \%-99.7 \%$ and $85.2 \%-94.9 \%$, varying depending on the reference population size, and a $4.7 \%-14.1 \%$ accuracy difference. Therefore, as the marker density or reference population size increases, the difference in imputation accuracy decreases, even if the frequency of minor alleles increases. There was a clear distinction among scenarios when the imputation accuracy threshold was $85 \%$. If a $15 \mathrm{k}$ marker density was used in all scenarios, the accuracy exceeded the threshold value.

\section{DISCUSSION}

In this study, we use Hanwoo genotype dataset from BioGreen 21 data set of National Institute

(A)

(B)
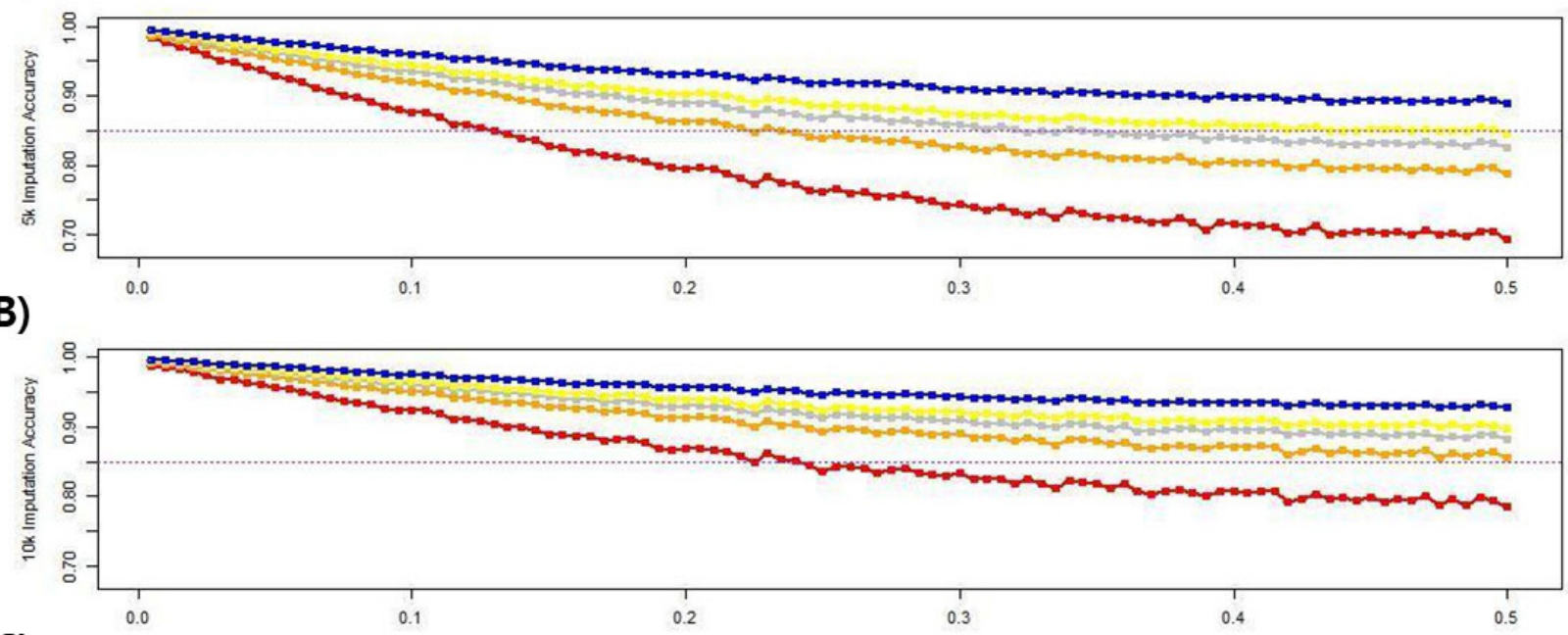

(C)

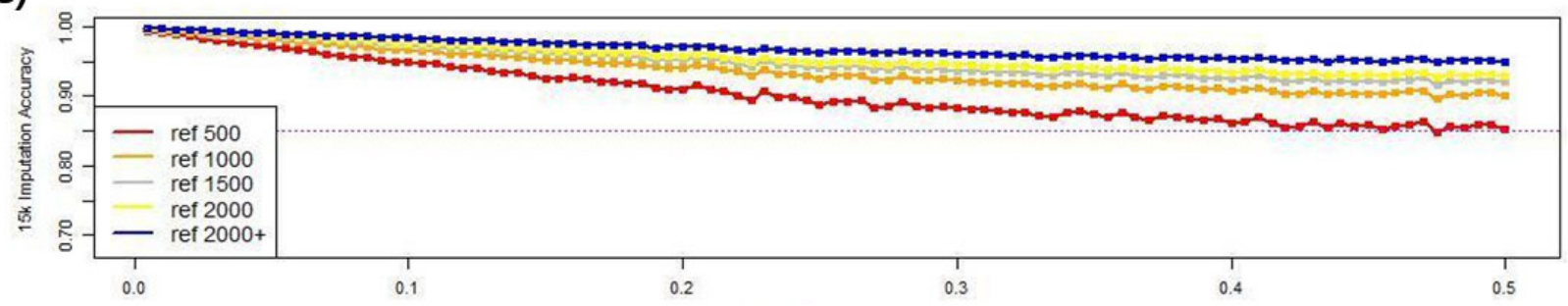

Fig. 11. The average accuracy of imputation according to marker density and minor allele frequency and reference population. The minor allele frequency was divided into 100 groups between 0 and 0.5 by 0.005 , and then calculated the average accuracy of SNPs belonging to the group (A) $5 \mathrm{k}$, (B) $10 \mathrm{k}$ and (C) $15 \mathrm{k}$. SNP, single nucleotide polymorphism. 
of Animal Science, RDA, to generate scenario data for genotype imputation. The validation data were the data set of the youngest 889 animals; and these were divided into four validation sets. The others were used as a reference group to perform imputation using Beagle 3.3, a population-based method.

The imputation accuracy was examined by direct comparison between the true and imputed genotypes. We investigated LD in Hanwoo cattle because the population LD level affects the imputation accuracy. Uemeto et al. (2015) confirmed that Japanese black cattle had 0.1 LD $\left(r^{2}\right)$ when there was $200 \mathrm{~kb}$ between SNP pairs [27]. Using 16 Holstein breeds, Hoze et al. (2013) reported 0.2 LD $\left(r^{2}\right)$ when there was $100 \mathrm{~kb}$ between SNP pairs [28]. Thus, high LD means that the association between SNP markers is also high. This increases the probability of appropriate inference for closely located SNPs during imputation. Hanwoo cattle have a lower $L D$ value than other cattle breeds and require a larger reference population to achieve high imputation accuracy.

Imputation accuracy of $95 \%$ in Japanese black cattle was obtained with reference populations greater than 400 [27]. In comparison, 90\% imputation accuracy was obtained in Holstein cattle with reference populations greater than 300, and 95\% imputation accuracy in Fleckvieh cattle with reference populations greater than 400 [29]. In Hanwoo cattle, the imputation accuracy was $88 \%$ at low-density (5k) for reference populations greater than 1,000, while it was the same as in Holsteins (where long chromosomes have greater imputation accuracy than short ones) [30].

Imputation accuracy is also influenced by the marker density of the validation data. In dairy cattle, the imputation accuracy of a reference population of 2,406 was $72 \%, 82 \%, 91 \%, 93 \%$, and $97 \%$ at marker densities of $384,768,1,536,2,480$, and 6,177 , respectively [31]. In this study, comparing the results of three low-density panels $(5 \mathrm{k}, 10 \mathrm{k}$, and $15 \mathrm{k})$, the accuracy differed by up to $13.6 \%$ according to the marker density. We need to assess imputation accuracy according to the reference population size because, as the population size increases, the haplotype data increase along with the explanatory power of each haplotype, and the imputation error rate decreases. We assessed imputation accuracy according to five reference population sizes, to determine the effect of reference population size on imputation accuracy in Hanwoo cattle. When the imputation was performed with a reference population over 2,000 (3,600), the accuracy was $93 \%$ at the lowest density $(5 \mathrm{k})$, which is lower than in other breeds.

The minor allele frequency also negatively affects the imputation accuracy. Because imputation imputes missing values through a statistical method, a correct genotype is accidentally introduced more often at a low minor allele frequency [27,32]. However, as the marker density or reference population size increases, the difference in imputation accuracy decreases, even if the frequency of minor alleles increases.

In conclusion, the imputation accuracy difference was $6.3 \%-13.6 \%$ among marker densities, varying depending on the reference population size, and $4 \%-11.3 \%$ among reference population sizes, varying according to marker density. In Hanwoo cattle, a reference population of at least 1,000 is needed to obtain more than $88 \%$ imputation accuracy.

\section{REFERENCES}

1. Meuwissen THE, Hayes BJ, Goddard ME. Prediction of total genetic value using genome-wide dense marker maps. Genetics. 2001;157:1819-29. https://doi.org/10.1093/genetics/157.4.1819

2. Hayes BJ, Visscher PM, Goddard ME. Increased accuracy of artificial selection by using the realized relationship matrix. Genet Res. 2009;91:47-60. https://doi.org/10.1017/ S0016672308009981 
3. Lande R, Thompson R. Efficiency of marker-assisted selection in the improvement of quantitative traits. Genetics. 1990;124:743-56. https://doi.org/10.1093/genetics/124.3.743

4. Zheng HJ, Wu AZ, Zheng CC, Wang YF, Cai R, Shen XF, et al. QTL mapping of maize (Zea mays) stay-green traits and their relationship to yield. Plant Breed. 2009;128:54-62. https://doi. org/10.1111/j.1439-0523.2008.01529.x

5. Zhang Z, Ding X, Liu J, Zhang Q, de Koning DJ. Accuracy of genomic prediction using low-density marker panels. J Dairy Sci. 2011;94:3642-50. https://doi.org/10.3168/jds.20103917

6. Wang Q, Yu Y, Yuan J, Zhang X, Huang H, Li F, et al. Effects of marker density and population structure on the genomic prediction accuracy for growth trait in Pacific white shrimp Litopenaeus vannamei. BMC Genet. 2017;18:45. https://doi.org/10.1186/s12863-017-0507-5

7. Habier D, Fernando RL, Dekkers JCM. Genomic selection using low-density marker panels. Genetics. 2009;182:343-53. https://doi.org/10.1534/genetics.108.100289

8. Meuwissen THE. Accuracy of breeding values of 'unrelated' individuals predicted by dense SNP genotyping. Genet Sel Evol. 2009;41:35. https://doi.org/10.1186/1297-9686-41-35

9. Weigel KA, de los Campos G, González-Recio O, Naya H, Wu XL, Long N, et al. Predictive ability of direct genomic values for lifetime net merit of Holstein sires using selected subsets of single nucleotide polymorphism markers. J Dairy Sci. 2009;92:5248-57. https://doi. org/10.3168/jds.2009-2092

10. Cheung CYK, Thompson EA, Wijsman EM. GIGI: an approach to effective imputation of dense genotypes on large pedigrees. Am J Hum Genet. 2013;92:504-16. https://dx.doi. org/10.1016/j.ajhg.2013.02.011

11. Saad M, Wijsman EM. Power of family-based association designs to detect rare variants in large pedigrees using imputed genotypes. Genet Epidemiol. 2014;38:1-9. https://doi. org/10.1002/gepi.21776

12. Marchini J, Howie B, Myers S, McVean G, Donnelly P. A new multipoint method for genome-wide association studies by imputation of genotypes. Nat Genet. 2007;39:906-13. https://doi.org/10.1038/ng2088

13. Howie BN, Donnelly P, Marchini J. A flexible and accurate genotype imputation method for the next generation of genome-wide association studies. PLOS Genet. 2009;5:e1000529. https://doi.org/10.1371/journal.pgen.1000529

14. O’Connell J, Gurdasani D, Delaneau O, Pirastu N, Ulivi S, Cocca M, et al. A general approach for haplotype phasing across the full spectrum of relatedness. PLOS Genet. 2014;10:e1004234. https://doi.org/10.1371/journal.pgen.1004234

15. Hickey JM, Kinghorn BP, Cleveland MA, Tier B, van der Wer JHJ. Recursive long range phasing and long haplotype library imputation: building a global haplotype library for Holstein cattle. In: Proceedings of the World Congress on Genetics Applied to Livestock Production; 2010; Leipzig, Germany. p. 0944.

16. Hickey JM, Crossa J, Babu R, de los Campos G. Factors affecting the accuracy of genotype imputation in populations from several maize breeding programs. Crop Sci. 2012;52:654-63. https://doi.org/10.2135/cropsci2011.07.0358

17. Scheet P, Stephens M. A fast and flexible statistical model for large-scale population genotype data: applications to inferring missing genotypes and haplotypic phase. Am J Hum Genet. 2006;78:629-44. https://doi.org/10.1086/502802

18. Browning SR, Browning BL. Rapid and accurate haplotype phasing and missing-data inference for whole-genome association studies by use of localized haplotype clustering. Am J Hum Genet. 2007;81:1084-97. https://doi.org/10.1086/521987 
19. Howie B, Fuchsberger C, Stephens M, Marchini J, Abecasis GR. Fast and accurate genotype imputation in genome-wide association studies through pre-phasing. Nat Genet. 2012;44:9559. https://doi.org/10.1038/ng.2354

20. VanRaden PM. Genomic evaluations with many more genotypes and phenotypes. In: Proceedings of the World Congress on Genetics Applied to Livestock Production; 2010; Leipzig, Germany. p. 0027.

21. Sambrook J, Fritsch EF, Maniatis T. Molecular cloning: a laboratory manual. New York: Cold Spring Harbor Laboratory Press; 1989.

22. Purcell S, Neale B, Todd-Brown K, Thomas L, Ferreira MAR, Bender D, et al. PLINK: a tool set for whole-genome association and population-based linkage analyses. Am J Hum Genet. 2007;81:559-75. https://doi.org/10.1086/519795

23. $\mathrm{R}$ Core Team. R: a language and environment for statistical computing [Internet]. $R$ Foundation for Statistical Computing. 2016 [cited 2021 Aug 7]. https://www.R-project.org/

24. Hill WG, Robertson A. Linkage disequilibrium in finite populations. Theor Appl Genet. 1968;38:226-31. https://doi.org/10.1007/BF01245622

25. Browning BL, Yu Z. Simultaneous genotype calling and haplotype phasing improves genotype accuracy and reduces false-positive associations for genome-wide association studies. Am J Hum Genet. 2009;85:847-61. https://doi.org/10.1016/j.ajhg.2009.11.004

26. Browning SR, Weir BS. Population structure with localized haplotype clusters. Genetics. 2010;185:1337-44. https://doi.org/10.1534/genetics.110.116681

27. Uemoto Y, Sasaki S, Sugimoto Y, Watanabe T. Accuracy of high-density genotype imputation in Japanese Black cattle. Anim Genet. 2015;46:388-94. https://doi.org/10.1111/age.12314

28. Hozé C, Fouilloux MN, Venot E, Guillaume F, Dassonneville R, Fritz S, et al. High-density marker imputation accuracy in sixteen French cattle breeds. Genet Sel Evol. 2013;45:33. https://doi.org/10.1186/1297-9686-45-33

29. Pausch H, Aigner B, Emmerling R, Edel C, Götz KU, Fries R. Imputation of high-density genotypes in the Fleckvieh cattle population. Genet Sel Evol. 2013;45:3. https://doi. org/10.1186/1297-9686-45-3

30. Erbe M, Hayes BJ, Matukumalli LK, Goswami S, Bowman PJ, Reich CM, et al. Improving accuracy of genomic predictions within and between dairy cattle breeds with imputed high-density single nucleotide polymorphism panels.J Dairy Sci. 2012;95:4114-29. https://doi. org/10.3168/jds.2011-5019

31. Chen L, Li C, Sargolzaei M, Schenkel F. Impact of genotype imputation on the performance of GBLUP and Bayesian methods for genomic prediction. PLOS ONE. 2014;9:e101544. https://doi.org/10.1371/journal.pone.0101544

32. Zhang Z, Xiao X, Zhou W, Zhu D, Amos CI. False positive findings during genome-wide association studies with imputation: influence of allele frequency and imputation accuracy. Hum Mol Genet. 2021:ddab203. https://doi.org/10.1093/hmg/ddab203 